\title{
The effectiveness of intravenous iron for iron deficiency anemia in gastrointestinal cancer patients: a retrospective study
}

\author{
Laurens Verhaeghe ${ }^{a}$, Luk Bruyneel ${ }^{b}$, Elisabeth Stragierc, Marc Ferrantec, Daan Dierickx ${ }^{d}$, Hans Prenen ${ }^{c}$
}

University Hospitals Leuven; Institute for Healthcare Policy, Leuven, Belgium

Abstract

${ }^{a}$ Department of Internal Medicine, University Hospitals Leuven (Laurens Verhaeghe); 'Institute for Healthcare Policy (Luk Bruyneel); 'Department of Gastroenterology and Hepatology, University Hospitals Leuven (Elisabeth Stragier, Marc Ferrante, Hans Prenen); dDepartment of Hematology, University Hospitals Leuven (Daan Dierickx), KU Leuven, Belgium

Conflict of Interest: Hans Prenen has received speaker fees from Vfor Pharma. Marc Ferrante has received personal fees and/or non-financial support from Takeda, Abbvie, Boehringer-Ingelheim, Chiesi, Falk, Ferring Janssen, Mitsubishi Tanabe, MSD, Tillotts and Zeria. The other authors disclose no conflict of interest. The authors have full control of all primary data and agree to allow the journal to review their data if requested

Funding: Hans Prenen is supported by the Belgian Foundation against Cancer. Marc Ferrante is a Senior Clinical Investigator of the Research Foundation - Flanders (FWO), Belgium

Correspondence to: Hans Prenen, MD PhD, University Hospitals Leuven, Department of Gastroenterology and Hepatology, Digestive Oncology Unit, Herestraat 49, B3000 Leuven, Belgium,

e-mail: hans.prenen@uzleuven.be

Received 14 June 2017; accepted 3 August 2017; published online 6 September 2017

DOI: https://doi.org/10.20524/aog.2017.0189

\section{Introduction}

Anemia is a common hematological side effect in patients with cancer, both at the time of diagnosis and during antineoplastic treatment. Some of the most important causes of anemia in this setting are blood loss, chemotherapyinduced myelosuppression and iron deficiency (ID), defined as either a decrease in the total iron content in the body or as a failure to release iron at a sufficient rate to keep pace with the demands of the bone marrow [1]. Patients with anemia have a poor prognosis, physical symptoms and a low quality of life; they therefore require appropriate treatment $[2,3]$. Research has shown that the effect of red blood cell (RBC) transfusion in cancer patients is temporary, while it may be associated with serious thromboembolic events and increased mortality $[4,5]$. Other treatment options include administration of erythropoietin-stimulating agents (ESA) and iron supplementation. However, only $38.9 \%$ of cancer patients have their anemia treated and only $6.5 \%$ are treated with iron supplementation alone [6]. 
According to a Cochrane review [7], the use of ESA in cancer-related anemia is beneficial in some aspects. These agents are, however, linked to severe adverse effects, such as increased mortality. Importantly, the proportion of patients reaching target hemoglobin $(\mathrm{Hb})$ levels is variable [8]. Recent guidelines on the treatment of cancer-related anemia therefore strongly recommend the reduction or prevention of transfusions and a restricted use of ESA at the lowest required dose [1,9].

The most important reason for ESA-refractory anemia is ID [10], which occurs in 43\% of cancer patients [11]. A recent Cochrane review evaluated ESA treatment in combination with iron supplementation [12]. The authors concluded that the addition of iron to ESA offers a superior hematopoietic response, reduces the need for $\mathrm{RBC}$ transfusions, improves $\mathrm{Hb}$ levels (mean difference $0.48 \mathrm{~g} / \mathrm{dL}$ ), and appears to be well tolerated in comparison to ESA alone. In addition, intravenous (IV) iron was found to be superior to oral iron supplementation in terms of hematopoietic response and mean change in $\mathrm{Hb}$ levels. This evidence supports the important and possibly underestimated role of IV iron administration on its own in the treatment of ID anemia in cancer patients [13]. A handful of studies also suggest that patients without concomitant ESA can benefit from IV iron therapy (Table 1). This includes three randomized controlled clinical trials showing that IV iron alone significantly reduces transfusion requirements in patients with gynecological cancers [14-16]. Two large observational studies also found that median $\mathrm{Hb}$ increases were not substantially different in patients treated with IV iron with or without ESA $[17,18]$. Finally, one small $(n=17)$ randomized controlled trial studied the effect of IV iron in patients suffering from anemia of chronic disease and also noted a significant change in $\mathrm{Hb}$ [19]. Also note that the use of pre- or perioperative intravenous iron therapy in oncological colorectal surgery might improve postoperative $\mathrm{Hb}$ levels and might decrease the need for postoperative RBC transfusion. However, firm conclusions on this issue have not yet been drawn [20,21].

This was a retrospective study of digestive oncology (DIO) patients receiving IV iron as monotherapy for ID anemia. We aimed to further evaluate the effect of this therapy on $\mathrm{Hb}$, transferrin saturation (TSAT) and serum ferritin (SF) levels. In addition, we performed a review of the pathophysiology of ID anemia in an oncology setting, paying specific attention to the role of hepcidin as an emerging parameter in differentiating different types of anemia in this population $[22,23]$. The main goal of this study was to identify the patients who benefit most from IV iron treatment.

\section{Patients and methods}

\section{Patients and study data}

We performed a retrospective observational study among patients with malignant disease of the gastrointestinal tract who were treated with intravenous ferric carboxymaltose (FCM) because of anemia. The study protocol was approved by the Ethics Committee of the University Hospitals Leuven.
Between 2012 and 2014, 513 patients were seen in the outpatient DIO clinic or were admitted to hospital. They had received a total of 619 intravenous FCM administrations. $\mathrm{Hb}$, TSAT and SF levels were recorded at baseline, which was on the day of FCM administration, and four weeks after the last administration. Not all parameters were available for all patients. Most patients only received one dose of FCM. If patients were administered a second dose within four weeks of the first administration, the two doses were considered as one cumulative dose. Patients' age, sex, body weight, type of primary tumor, tumor staging, type of chemotherapy (if applicable), and cumulative dose of FCM were also documented.

According to the standard of care FCM-dosing scheme (Table 2), based upon the modified Ganzoni formula, which estimates a patient's total body iron deficit using body weight and baseline $\mathrm{Hb}$ [24], patients were grouped as follows: those who were treated adequately, and those who were treated inadequately (insufficient dose of FCM according to body weight and baseline $\mathrm{Hb}$ ); patients were also grouped according to low or high baseline ferritin levels. As SF levels $<100 \mu \mathrm{g} / \mathrm{L}$ in combination with TSAT $<20 \%$ strongly suggest absolute ID (AID), whereas SF levels $>100 \mu \mathrm{g} / \mathrm{L}$ and TSAT $<20 \%$ usually indicate iron sequestration as seen in anemia of chronic disease (ACD), a cutoff point of $\mathrm{SF}=100 \mu \mathrm{g} / \mathrm{L}$ was chosen [25].

\section{Statistical analysis}

Quantitative variables were expressed as mean \pm standard deviation (STD), as median value and full range (minimummaximum), or as median value and interquartile range (IQR). The Mann-Whitney $U$ test was used to compare variables between two independent groups (adequate vs. non-adequate, low baseline SF vs. high baseline SF). The Wilcoxon signed-rank test was used to compare variables within one group (baseline vs. follow up). A P-value $<0.05$ was considered statistically significant. The data analysis for this paper was generated using SAS software, Version 9.4 M4 of the SAS System for Windows (SAS Institute Inc., Cary, NC, USA).

\section{Results}

Of the 513 initially identified patients, 99 were excluded because they lacked information essential for analysis (i.e. baseline or follow-up $\mathrm{Hb}$ was not retrieved). Forty-one (9.9\%) of the remaining 414 patients received a transfusion with RBC, despite FCM treatment at baseline or during the follow-up period, and were excluded from the analysis. In addition, 54 of the remaining 373 patients were excluded because their baseline iron status was unknown. We also excluded patients who had either a baseline TSAT $>20 \%$ $(\mathrm{n}=14)$ or a baseline SF $>30 \mu \mathrm{g} / \mathrm{L}$ and no baseline TSAT $(\mathrm{n}=2)$, as the other patients seemed most likely to be iron deficient (functional or absolute) [25] and since this subgroup appeared too small for analysis. 
IV iron in anemic GI cancer patients 3

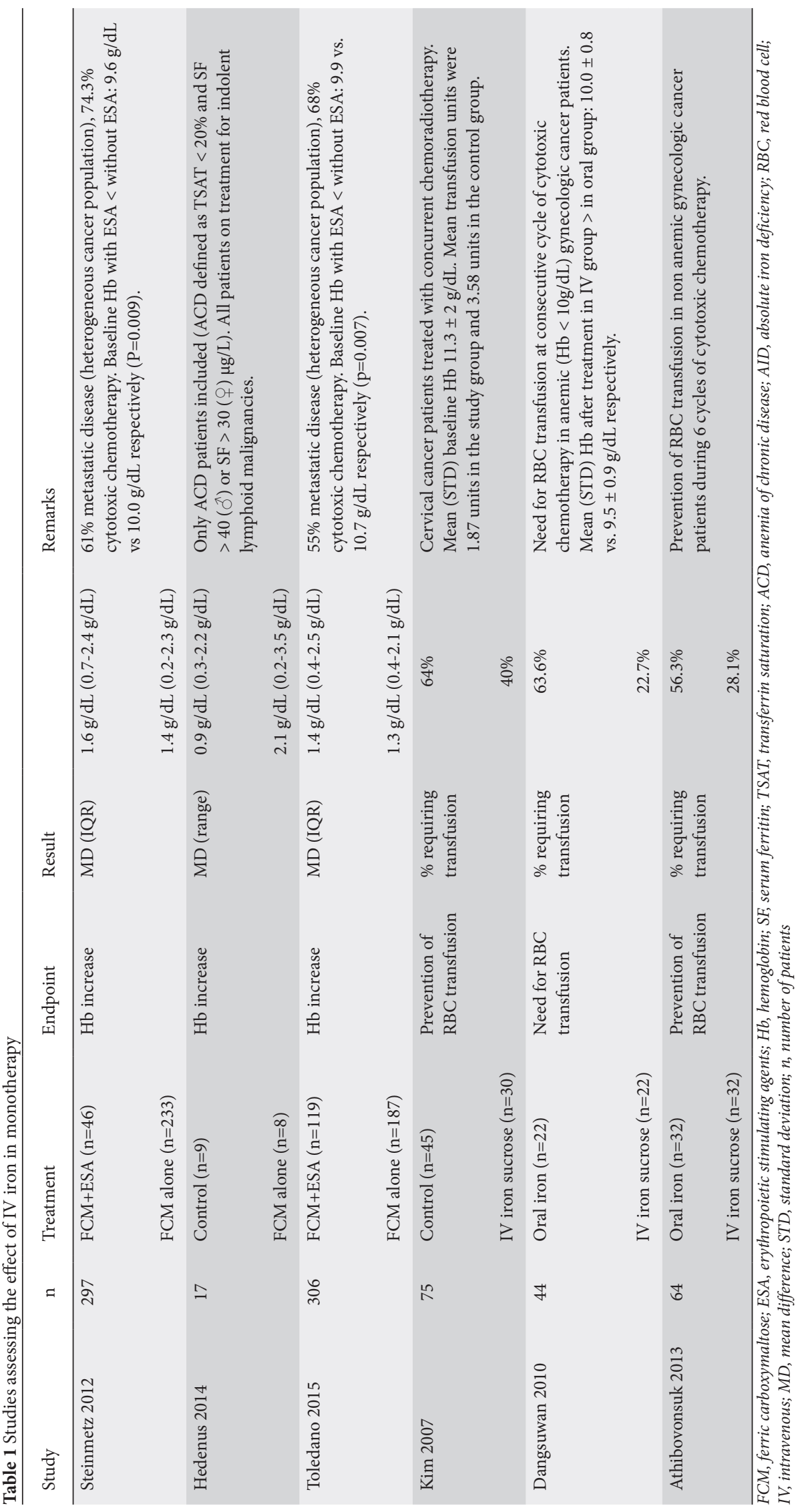


Table 2 Ferric carboxymaltose dosing scheme

\begin{tabular}{lcc}
$\mathrm{Hb}(\mathrm{g} / \mathrm{dL})$ & Body weight 50 to $<70 \mathrm{~kg}$ & Body weight $\geq 70 \mathrm{~kg}$ \\
\hline$<10$ & $1500 \mathrm{mg}$ & $2000 \mathrm{mg}$ \\
$\geq 10$ & $1000 \mathrm{mg}$ & $1500 \mathrm{mg}$ \\
\hline
\end{tabular}

Patients weighing $<50 \mathrm{~kg}$ received a maximum dosage of $20 \mathrm{mg} / \mathrm{kg}$

Hence, data for 303 patients were retained for analytic purposes. None of these patients received ESA; 57 (18.8\%) of them received two separate doses of FCM within four weeks.

\section{Patient characteristics (Table 3)}

The median (IQR) age of our population was 64 (55-72) years. About $75 \%(n=226)$ had metastatic disease and $90.1 \%$ $(n=273)$ were on active myelosuppressive chemotherapy. The median dose of FCM administered was $1000 \mathrm{mg}$.

One hundred nineteen patients were treated adequately (39.3\%) and 184 were treated inadequately (60.7\%). No significant differences in other demographics or disease characteristics were noted between these subgroups.

Baseline and follow-up $\mathrm{Hb}$ levels were available for the whole study group $(n=303)$. Iron status parameters were assessed in 52\% (SF, $\mathrm{n}=157)$ and $99 \%$ (TSAT, $\mathrm{n}=301$ ) of patients at baseline. Follow-up values were available for $31 \%(\mathrm{SF}, \mathrm{n}=95)$ and $64 \%$ (TSAT, $n=193$ ) of patients. However, both baseline and follow-up values were retrieved in only $23 \%$ (SF, $n=69)$ and $63 \%$ (TSAT, $n=192$ ) of patients.

\section{Effectiveness: overall group (Table 4)}

The median (IQR) baseline levels of $\mathrm{Hb}, \mathrm{SF}$ and TSAT were 10.0 (9.2-10.9) g/dL, 219 (47-610) $\mu \mathrm{g} / \mathrm{L}$ and $12(8-14) \%$, respectively. At follow up, $\mathrm{Hb}$ was 10.8 (9.6-11.9) g/dL, SF was $988(528-1715) \mu \mathrm{g} / \mathrm{L}$ and TSAT was $22(16-29) \%$. The differences were 0.5 (-0.1-1.6) g/dL for $\mathrm{Hb}, 464$ (230-830) $\mu \mathrm{g} / \mathrm{L}$ for SF and 11 (5-18.5) \% for TSAT. All these increases were statistically significant $(\mathrm{P}<0.001)$.

\section{Effectiveness: adequately vs. inadequately dosed subgroups (Table 4)}

A statistically significant difference was observed in baseline $\mathrm{Hb}$ between the inadequately and the adequately dosed group: 9.7 (9.0-10.7) vs. 10.5 (9.5-11.2) g/dL, respectively $(\mathrm{P}<0.001)$. The levels at follow up were also significantly different, with the inadequately dosed group ending up with a lower followup Hb level: median (IQR) Hb, 10.7 (9.6-11.7) vs. 11.2 (9.9$12.2) \mathrm{g} / \mathrm{dL}(\mathrm{P}=0.036)$. There was a significant increase in $\mathrm{Hb}$ from baseline to follow up in both subgroups $(\mathrm{P}<0.001)$. The difference between the median changes in the two subgroups, however, was not significant $(\mathrm{P}=0.206)$.

Iron parameters did not differ significantly between these subgroups at baseline or at follow up, and the same was observed for the median change seen in these groups.

\section{Effectiveness: low baseline ferritin vs. high baseline ferritin subgroups (Table 4)}

Fifty patients started with a low level of SF and 107 patients had an SF level higher than $100 \mu \mathrm{g} / \mathrm{L}$ at baseline. Baseline $\mathrm{Hb}$ levels were statistically significantly different across subgroups, with a median baseline (IQR) level of $10.1(9.2-11.3) \mathrm{g} / \mathrm{dL}$ in the low-SF group and 9.7 (8.7-10.7) $\mathrm{g} / \mathrm{dL}$ in the high-SF group $(\mathrm{P}=0.044)$. Baseline TSAT levels were statistically significantly lower in the low-SF group: 7.5 (5-11) vs. 13 (10-14) \% in the high-SF group $(\mathrm{P}<0.001)$. In the low-ferritin group, $42 \%$ were treated adequately; in the high ferritin group, $30 \%$ were treated adequately.

At follow up, median (IQR) Hb levels were also statistically significantly different, with 11.7 (10.3-12.6) g/dL in the low-SF group and $10.6(9.2-11.5) \mathrm{g} / \mathrm{dL}$ in the high-SF group $(\mathrm{P}<0.001)$. Median SF levels at follow up were also different $(\mathrm{P}=0.001)$. However, median (IQR) TSAT levels were similar: 24 (16-31) $\%$ in the low baseline SF group vs. 20 (15-28) \% in the high baseline SF group, $(\mathrm{P}=0.414)$.

In the low baseline SF group, the median (IQR) change in $\mathrm{Hb}$ was significantly higher than in the high baseline SF group: $1.2(0.3-2.2)$ vs. $0.4(-0.3-1.4) \mathrm{g} / \mathrm{dL}$, respectively $(\mathrm{P}=0.004)$. The increase in TSAT was also significantly greater in the low-SF group: 18 (8-22) vs. 8 (0-17) \% for the high-SF group $(\mathrm{P}=0.007)$. The SF increase was similar in both groups.

\section{Discussion}

In general, three ID syndromes leading to ironrestricted erythropoiesis and therefore ID anemia can be differentiated: AID; functional ID (FID); and iron sequestration in ACD [26]. All three of these mechanisms can play a role in cancer patients [8]. FID occurs when bone marrow requirements are higher than iron stores. AID is the absence of storage iron, which can occur through nutritional deficiencies, blood loss or malabsorption. Finally, ACD with iron sequestration occurs in a state of inflammation where hepcidin acts to sequester iron by inhibiting the egress of storage iron from hepatocytes and macrophages into plasma, and also by inhibiting the absorption of dietary iron in duodenal enterocytes [27]. It is well known that interleukin (IL)-6, among other cytokines, readily induces hepcidin release from hepatocytes. Since IL- 6 is abundant in inflammatory states such as cancer, this explains the important role of hepcidin in ACD.

Differentiating between these three ID types is important, since current National Comprehensive Cancer Network guidelines [1] recommend IV iron for AID patients, and ESA in addition to IV iron for ACD patients. However, making the differential diagnosis with the current available tests remains challenging. In addition, multiple mechanisms tend to occur simultaneously, leading to a mixed status of ID (e.g., AID coexisting with ACD) [26]. Conventional iron status parameters are combined to differentiate between AID and ACD [28], but all of these parameters have disadvantages, 


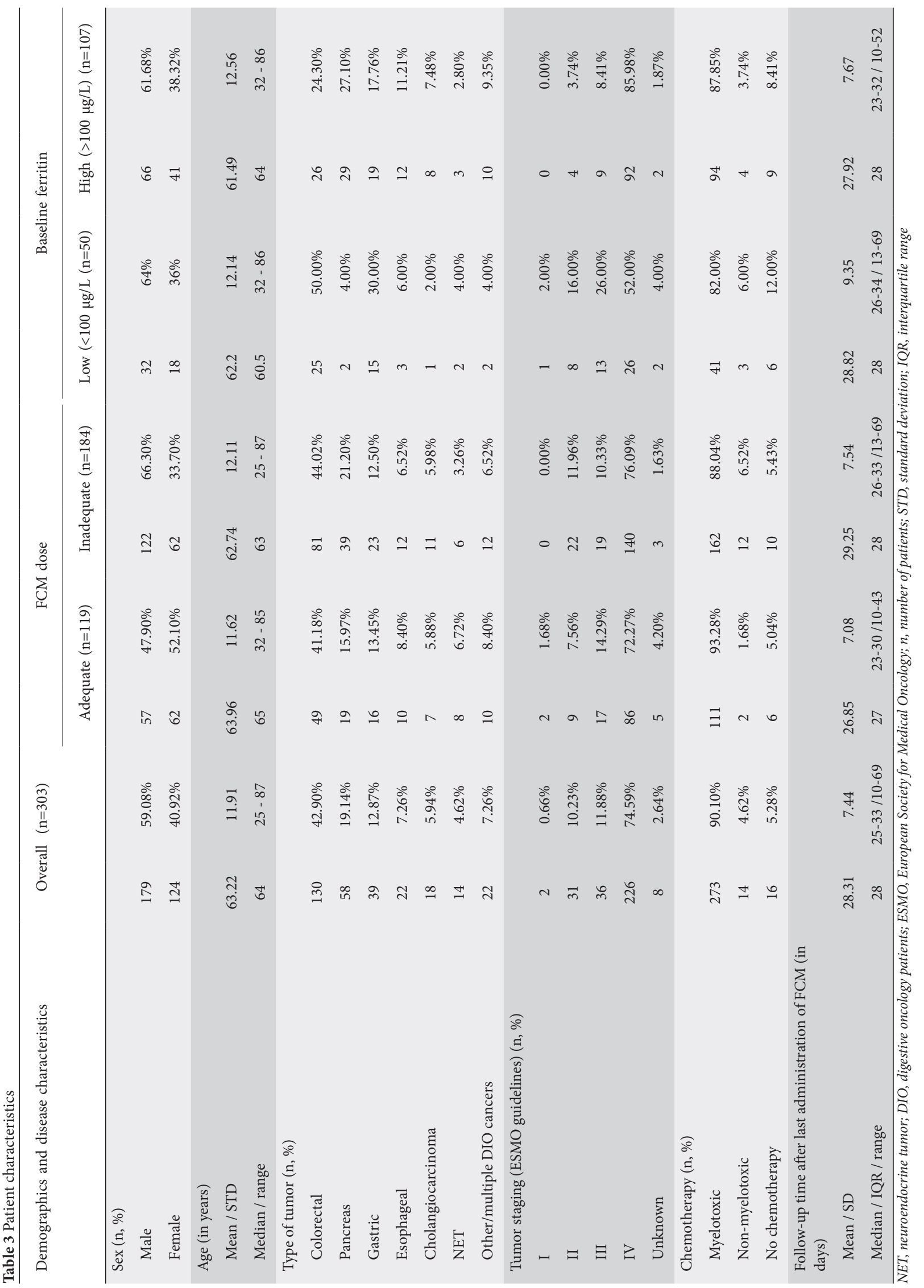




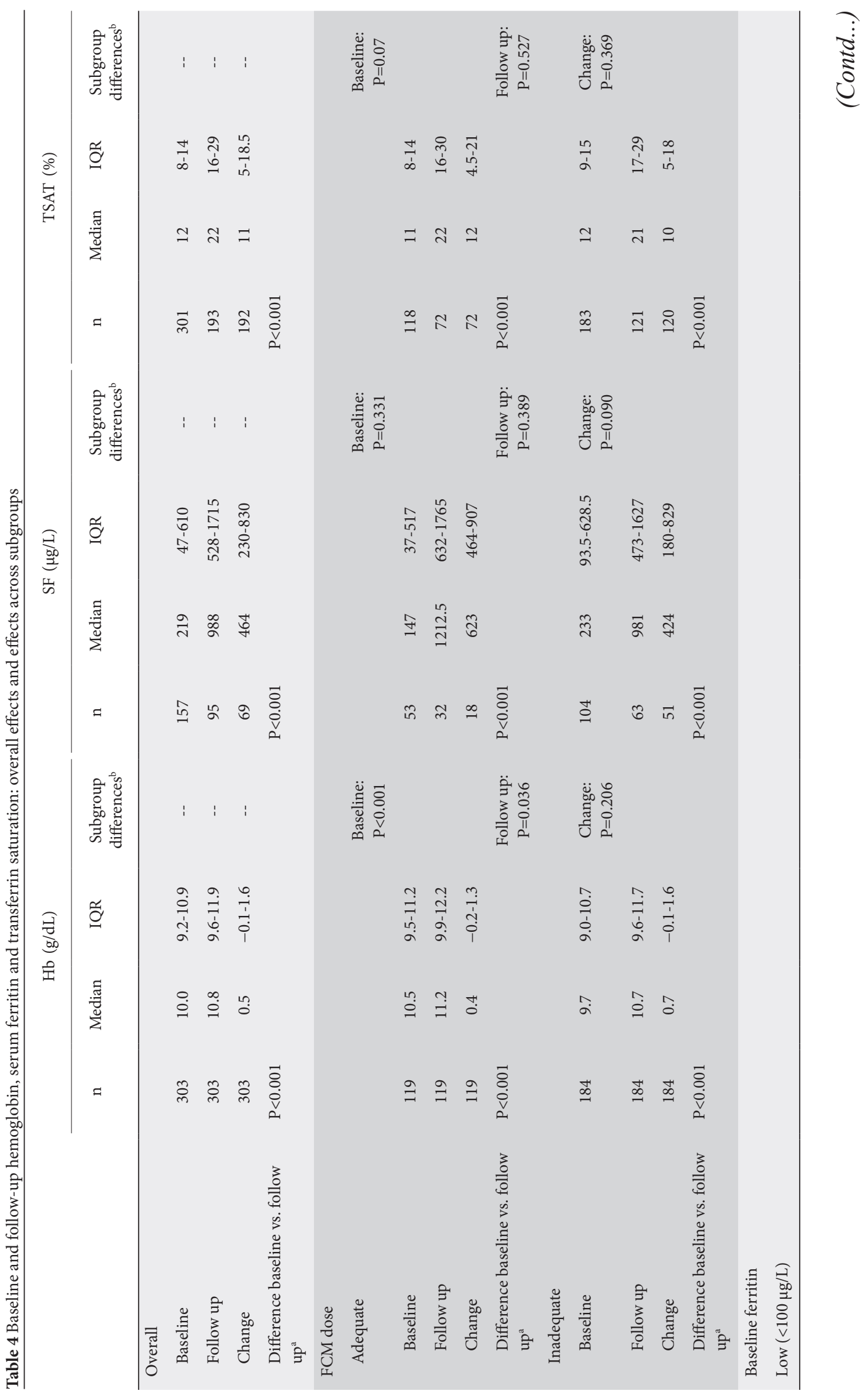




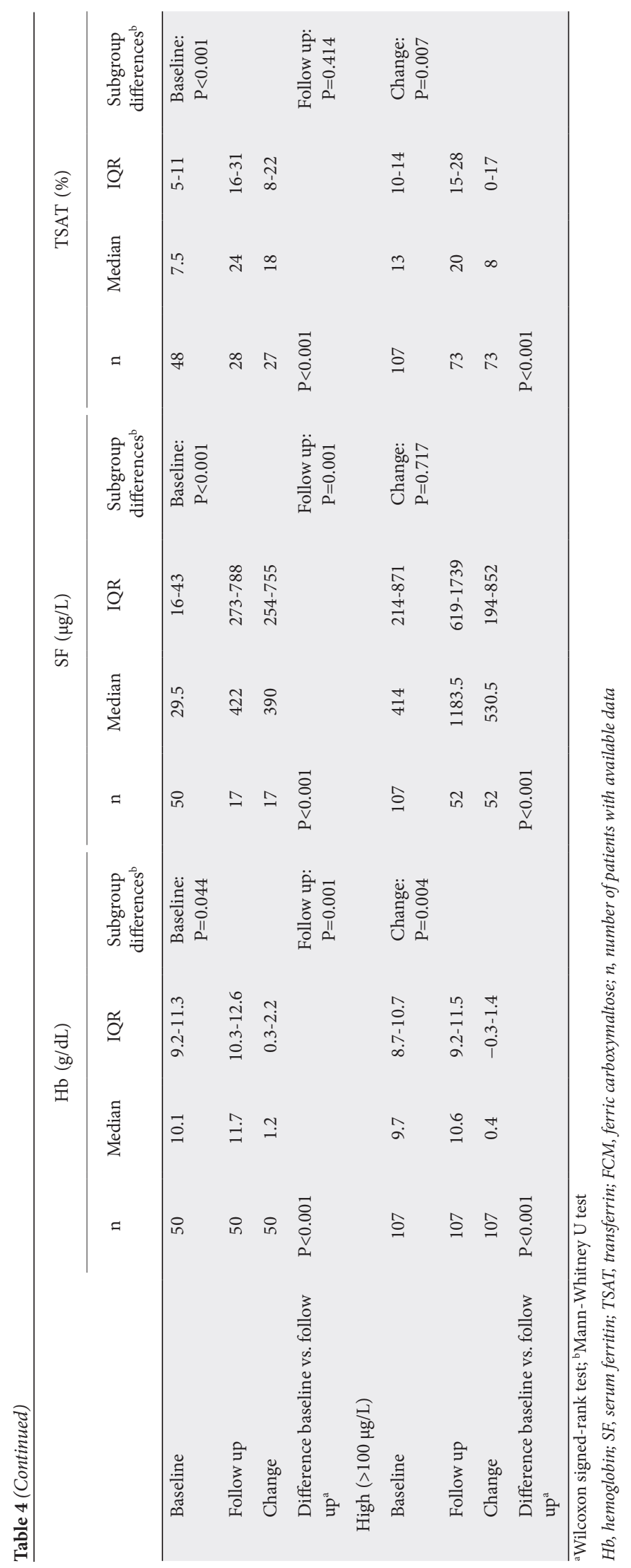


with substantial overlap between normal subjects, AID, and ACD patients. One of the main problems is that SF is also an acute-phase protein, which is often elevated in inflammatory states (e.g., cancer) and therefore cannot be used reliably as a reflection of iron stores. Furthermore, cutoff values of SF and TSAT to differentiate between ACD and AID differ between studies and often seem to be chosen arbitrarily.

In recent years, hepcidin levels have been proposed as a diagnostic tool, since changes in hepcidin concentrations, in contrast to changes in SF levels, are the cause rather than the result of iron disorders. Shu et al [23] divided 89 cancer patients into AID and ACD groups by Prussian blue staining of bone marrow smears (considered the gold standard diagnostic method to define whether a patient is iron deficient or not [29]) and demonstrated that hepcidin is a promising approach for the discrimination of tumor-related AID and ACD. They found that hepcidin expression showed a decreasing trend in the tumor-related ACD group, the control group and the tumor-related AID group. Furthermore, they identified high expression of IL-6 in both the AID and ACD groups compared with controls, suggestive of chronic inflammation. In the ACD group, IL-6 significantly increased hepcidin expression. In the AID group however, no correlation was found between IL-6 and hepcidin, which indicates that AID can relatively reduce hepcidin levels, even in the presence of inflammation.

One study so far has evaluated the predictive value of hepcidin levels on therapy outcome in patients with chemotherapy-associated anemia treated with ESA, with or without iron supplementation [22]. When the hepcidin level was lower than $64.3 \mathrm{ng} / \mathrm{mL}$ (lower two tertiles), the addition of high-dose IV iron to ESA seemed to result in a better clinical response in comparison to ESA alone, ESA with oral iron, ESA with placebo, or ESA with low-dose IV iron: none of the 47 patients in the ESA and high-dose IV iron groups required a transfusion, compared with a $14 \%$ transfusion rate for the remaining study population $(\mathrm{P}=0.0065)$. When the hepcidin level was higher than $64.3 \mathrm{ng} / \mathrm{mL}$ (upper tertile), 6 out of 35 patients required a transfusion and the addition of high- or low-dose IV iron to ESA offered no benefit compared with oral iron or placebo. The erythropoietic response rate was significantly better for the low-hepcidin group (92-95\%) than for the high-hepcidin group (56\%) when IV iron was added to ESA. Low hepcidin levels may therefore characterize those ID patients who are more likely to benefit from intensive IV iron supplementation. The failure of IV iron to augment erythropoiesis when hepcidin levels are high casts doubt on whether IV iron alone can routinely overcome ACD.

This retrospective observational study documents the effect of FCM treatment for ID anemia in a group of DIO patients. In order to assess the actual effect of FCM treatment, patients receiving RBC transfusion (9.9\%) were excluded. This is the first study to document the effect of IV iron as monotherapy in a large group of DIO patients. Furthermore, we differentiated between the effects of this treatment on AID and on ACD patients, assuming that an SF level lower than $100 \mu \mathrm{g} / \mathrm{L}$ correlates with AID, while a level higher than $100 \mu \mathrm{g} / \mathrm{L}$ indicates ACD. Although this approach may be too simple to differentiate appropriately, it can help shape research agendas.
For the total study group, the median increase in $\mathrm{Hb}$ was $0.5(-0.1-1.6) \mathrm{g} / \mathrm{dL}$ after a median (IQR) follow-up period of 28 days (25-33). The fact that no significant difference in therapy response was found between the adequate and the inadequate group might imply that current dosing schemes are not optimal. Firm conclusions cannot be drawn, because our two subgroups started with a different baseline $\mathrm{Hb}$. However, a review by Gozzard [30] in 2011 already stated that cumulative doses up to $3000 \mathrm{mg}$ of IV iron should be considered in cancer patients. The author also suggested that higher doses of IV iron may overcome impaired iron absorption associated with hepcidin blockage in ACD.

The median change in $\mathrm{Hb}$ levels in our study was lower than those reported in previous studies (Table 1). Since our inadequately-treated group started with a baseline $\mathrm{Hb}$ that was lower than the adequately-treated group, patients that had the highest need for treatment (i.e., those with the lower $\mathrm{Hb}$ levels at baseline) were actually undertreated. The fact that our population had a higher percentage of metastatic disease and a higher proportion of patients on active myelosuppressive chemotherapy than previous studies might be another explanation for this difference (Table 1 vs. Table 3). Finally, our subgroup of DIO patients might not respond in the same way to IV iron therapy as other cancer patients do. A reason for this might be that the high rate of liver metastases in this group impairs liver function capacity, which could also compromise iron homeostasis.

As previously stated, differentiating between ACD and AID with current laboratory testing is not easy in cancer patients. We considered our low baseline SF group as AID patients and our high baseline SF groups as ACD patients. A significant difference was found between these two groups: the low ferritin/AID group showed a better response to FCM treatment, since their median change in $\mathrm{Hb}$ was significantly better. This implies a difference in response to IV iron therapy depending on the mechanism of ID, with monotherapy IV iron benefiting AID patients more than those with ACD.

These findings support the studies by Shu et al [23] and Steensma et al [22]. The latter stated that low serum hepcidin levels may help predict the response to ESA and supplemental iron. Shu et al showed that low hepcidin levels correspond with AID. Moreover, this study stated that, even in an inflammatory state (such as cancer), hepcidin levels remain a reliable marker to differentiate between AID and ACD (in contrast to ferritin). Remarkably, the cutoff of serum hepcidin levels indicated a better therapy-response in the Steensma et al study, and the levels corresponding with AID in the Shu et al study were almost exactly the same: $<64.3 \mathrm{ng} / \mathrm{mL}$ and $<64.1 \pm 15.9 \mathrm{ng} / \mathrm{mL}$ (mean \pm STD), respectively.

As this was a retrospective study, several shortcomings were inevitable and therefore firm conclusions cannot be drawn. First, we did not have a control group in our analysis, nor did we evaluate the effect on transfusion requirements. We excluded patients receiving RBC transfusion from analyses, thereby possibly introducing a bias that favors treatment with FCM. The follow-up period to assess the response to FCM therapy might have been too short, and its full effect could thus 
have been underestimated. We did not assess kidney function or vitamin B12/folic levels as possible confounding factors, nor was our patients' surgical status known. Finally, we did not evaluate a possible benefit as regards long-term outcome in terms of survival, nor did we investigate the effect on quality of life.

In conclusion, IV iron monotherapy plays a role in ID anemia in DIO patients treated with myelosuppressive chemotherapy. An important finding is that the response to therapy is dependent on the underlying mechanism. At present, differentiating between AID and ACD is not easy. With hepcidin emerging as a possible marker for differentiating between ID subtypes, this might become more feasible in the future. As assessing hepcidin status becomes more available in modern laboratories, future studies should include this marker in the evaluation of anemia to further investigate its clinical implications in terms of predicting response to therapy.

\section{Summary Box}

\section{What is already known:}

- Anemia in digestive oncology patients is common and is often associated with iron deficiency (ID)

- The underlying mechanisms of ID anemia in oncology patients can differ. At present, differentiating between them (absolute ID anemia and anemia of chronic disease) is not easy

- Treatment of ID anemia with erythropoietic agents has been abandoned because of significant side effects

- IV iron as a monotherapy has only been evaluated in a handful of studies with very heterogeneous groups of oncology patients, but seems to be beneficial

\section{What the new findings are:}

- IV iron as a monotherapy in a subset of gastrointestinal cancer patients seems to be beneficial, although therapy response is dependent on the underlying mechanism of ID

- Baseline ferritin levels seem to be a good predictor of therapy response

- With hepcidin emerging as a possible marker for differentiating between anemia subtypes, this might become a more valuable predictor of therapy response in the future

\section{References}

1. Cancer- and chemotherapy-induced anemia. NCCN Guidelines Version 1.2018 - June 23, 2017.
2. Holzner B, Kemmler G, Greil R, et al. The impact of hemoglobin levels on fatigue and quality of life in cancer patients. Ann Oncol 2002;13:965-973.

3. Lind M, Vernon C, Cruickshank D, et al. The level of haemoglobin in anaemic cancer patients correlates positively with quality of life. Br J Cancer 2002;86:1243-1249.

4. Mercadante S, Ferrera P, Villari P, David F, Giarratano A, Riina S. Effects of red blood cell transfusion on anemia-related symptoms in patients with cancer. J Palliat Med 2009;12:60-63.

5. Khorana AA, Francis CW, Blumberg N, Culakova E, Refaai MA, Lyman GH. Blood transfusions, thrombosis and mortality in hospitalized cancer patients. Arch Intern Med 2008; 168:2377-2381.

6. Ludwig H, Van Belle S, Barrett-Lee P, et al. The European Cancer Anaemia Survey (ECAS): A large, multinational, prospective survey defining the prevalence, incidence, and treatment of anaemia in cancer patients. Eur J Cancer 2004;40:2293-2306.

7. Tonia T, Mettler A, Robert N, et al. Erythropoietin or darbepoetin for patients with cancer. Cochrane Database Syst Rev 2012;12:CD003407.

8. Verraes K, Prenen H. Iron deficiency in gastrointestinal oncology. Ann Gastroenterol 2015;28:19-24.

9. Rizzo JD, Brouwers M, Hurley P, et al; American Society of Hematology. American Society of Clinical Oncology/American Society of Hematology clinical practice guideline update on the use of epoetin and darbepoetin in adult patients with cancer. J Clin Oncol 2010;28:4996-5010.

10. Goodnough LT. Erythropoietin and iron-restricted erythropoiesis. Exp Hematol 2007;35:167-172.

11. Ludwig H, Müldür E, Endler G, Hübl W. Prevalence of iron deficiency across different tumors and its association with poor performance status, disease status and anemia. Ann Oncol 2013;24:1886-1892.

12. Mhaskar R, Wao H, Miladinovic B, Kumar A, Djulbegovic B. The role of iron in the management of chemotherapy-induced anemia in cancer patients receiving erythropoiesis-stimulating agents. Cochrane Database Syst Rev 2016;2:CD009624.

13. Ludwig H, Aapro M, Bokemeyer C, et al. A European patient record study on diagnosis and treatment of chemotherapy-induced anaemia. Support Care Cancer 2014;22:2197-2206.

14. Kim YT, Kim SW, Yoon BS, et al. Effect of intravenously administered iron sucrose on the prevention of anemia in the cervical cancer patients treated with concurrent chemoradiotherapy. Gynecol Oncol 2007; 105:199-204.

15. Dangsuwan P, Manchana T. Blood transfusion reduction with intravenous iron in gynecologic cancer patients receiving chemotherapy. Gynecol Oncol 2010;116:522-525.

16. Athibovonsuk P, Manchana T, Sirisabya N. Prevention of blood transfusion with intravenous iron in gynecologic cancer patients receiving platinum-based chemotherapy. Gynecol Oncol 2013;131:679-682.

17. Steinmetz T, Tschechne B, Harlin O, et al. Clinical experience with ferric carboxymaltose in the treatment of cancer- and chemotherapy-associated anaemia. Ann Oncol 2013;24:475-482.

18. Toledano A, Luporsi E, Morere JF, et al. Clinical use of ferric carboxymaltose in patients with solid tumours or haematological malignancies in France. Support Care Cancer 2016;24:67-75.

19. Hedenus M, Karlsson T, Ludwig H, et al. Intravenous iron alone resolves anemia in patients with functional iron deficiency and lymphoid malignancies undergoing chemotherapy. Med Oncol 2014;31:302.

20. Hallet J, Hanif A, Callum J, et al. The impact of perioperative iron on the use of red blood cell transfusions in gastrointestinal surgery: a systematic review and meta-analysis. Transfus Med Rev 2014;28:205-211.

21. Ng O, Keeler BD, Mishra A, et al. Iron therapy for pre-operative 
anaemia. Cochrane Database Syst Rev 2015;22:CD011588.

22. Steensma DP, Sasu BJ, Sloan JA, Tomita DK, Loprinzi CL. Serum hepcidin levels predict response to intravenous iron and darbepoetin in chemotherapy-associated anemia. Blood 2015;125:3669-3671.

23. Shu T, Jing C, Lv Z, Xie Y, Xu J, Wu J. Hepcidin in tumor-related iron deficiency anemia and tumor-related anemia of chronic disease: pathogenic mechanisms and diagnosis. Eur J Haematol 2015;94:67-73.

24. Koch TA, Myers J, Goodnough LT. Intravenous iron therapy in patients with iron deficiency anemia: dosing considerations. Anemia 2015;2015:763576.

25. Muñoz M, García-Erce JA, Remacha F. Disorders of iron metabolism. Part II: iron deficiency and iron overload. J Clin Pathol
2011;64:287-296.

26. Goodnough LT. Iron deficiency syndromes and iron-restricted erythropoiesis (CME). Transfusion 2012;52:1584-1592.

27. Ganz T. Hepcidin and iron regulation, 10 years later. Blood 2011;117:4425-4433.

28. Thomas C, Thomas L. Biochemical markers and hematologic indices in the diagnosis of functional iron deficiency. Clin Chem 2002;48:1066-1076.

29. Kenneth K, Marshall AL, Ernest B, Thomas JK, Uri S, Josef TP. Williams Hematology, $8^{\text {th }}$ ed. McGraw-Hill press: New York, NY; 2010, pp. 617-624.

30. Gozzard D. When is high-dose intravenous iron repletion needed? Assessing new treatment options. Drug Des Devel Ther 2011;5:51-60. 